\title{
NUMERICAL INVESTIGATION OF THE WALL PRESSURE FLUCTUATIONS IN CHANNEL FLOWS
}

\author{
U. SCHUMANN* \\ Gesellschaft für Kernforschung, Institut für Reaktorentwicklung, Karlsruhe, Germany
}

Received 24 October 1974

\begin{abstract}
Numerically simulated turbulent pressure fields in a plane channel and an annulus with a 5:1 ratio of radii are used to evaluate the spatial structure of pressure fluctuations at the walls. Wall-pressure correlation functions in the axial and azimuthal directions, spectra, correlation functions and angular correlations of the forces acting on the cylinders of the annulus are considered. It is found that no correlation exists between the pressure values at two points which are diametrically opposed to each other in the annulus. The spectrum of the forces starts with a zero value for small wave numbers, reaches its maximum at about unity wave number and then decreases, as a $-7 / 3$ rd power of the wave number.
\end{abstract}

\section{Introduction}

The turbulent flow of incompressible fluids in an annulus and a plane channel has been simulated by means of a finite difference scheme [1,2]. Here, the resultant three-dimensional and time-dependent pressure and velocity fields are used to investigate the spatial structure of the fluctuating pressure at the walls. The resultant forces acting on the inner and outer cylinders of the annulus can be found from these pressure fields by proper circumferential integration.

These forces are able to induce vibrations of the inner and outer cylinders. Such vibrations are expected for fuel rods in nuclear subassemblies and might cause serious damage. This problem has been studied by many authors [3-7]. Because of the need for an integrating device there seems to be no direct way of measuring the forces experimentally. This can be done very easily numerically once the pressure field at a specific time is known. The pressure fluctuations in the numerical model are the consequence of turbulent motion only. So we are not confronted with the prob-

\footnotetext{
* On sabbatical leave with the Advanced Study Program, National Center for Atmospheric Research, Boulder, Colorado 80303. The National Center for Atmospheric Research is sponsored by the National Science Foundation.
}

lem of discriminating the fluctuations caused by the experimental test loop [7]. In fact, the results presented here may help to distinguish between these types of pressure fluctuations. The main point to be proven in this paper is the feasibility of the numerical approach. Some limitations in accuracy do exist, however, as will be discussed below.

\section{Specification of the computer experiments}

We consider a plane channel and one concentric annulus with $R_{2} / R_{1}=5$ as the ratio of radii. The flow is assumed to be incompressible, highly turbulent and steady state in its statistics. The mean flow direction is axial. The coordinates are $\left\{x_{1}, x_{2}, x_{3}\right\}$ for the plane channel and $\{x, \varphi, r\}$ for the annulus; $x_{1}$ and $x$ are the axial, $x_{3}, r$ the cross-stream and $x_{2}, \varphi$ the azimuthal directions. All dimensional variables are scaled by the friction velocity $u_{0}=\left(\left\langle\tau_{\mathrm{w}_{0}}\right\rangle\right)^{1 / 2}$ and the distance $D=R_{2}-R_{1}$ between the walls. Here, $\left\langle\tau_{\mathbf{w}_{0}}\right\rangle$ is the algebraic mean of the timemean wall stresses $\left\langle\tau_{\mathbf{w}_{1}}\right\rangle$ and $\left\langle\tau_{\mathbf{w}_{2}}\right\rangle$ (each divided by density) at the inner and outer wall $\left(r=R_{1}, R_{2}\right)$ :

$\left\langle\tau_{\mathrm{w}_{0}}\right\rangle=\left(R_{1}\left\langle\tau_{\mathrm{w}_{1}}\right\rangle+R_{2}\left(\tau_{\mathrm{w}_{2}}\right)\right) /\left(R_{1}+R_{2}\right)$.

The Reynolds number defined by the distance $D$, the 
kinematic viscosity $\nu_{0}$ and the mean axial velocity is equal to $3 \times 10^{5}$. The viscous diffusion is negligible, except for a thin sublayer adjacent to the walls.

The method is a finite difference scheme as described in ref. [1]. It integrates the Navier-Stokes equations directly, giving the gross scale structure of the turbulent flow as defined by the resolution on a grid. The subgrid scale (SGS) motion not resolvable by the grid has to be described by some SGS theory $[1,8]$. This theory is based mainly on the assumption of local isotropy and Kolmogorov's energy spectrum. Since the power spectrum of the pressure fluctuations decreases as $k^{-7 / 3}$, where $k$ is the wave number [9], we neglect contributions of the SGS motion to the pressure fluctuations.

No-slip conditions are used at the wall and periodicity is assumed in the $x_{1}-x_{2}$-planes. Taking the divergence of the Navier-Stokes equations we obtain the following Poisson equation to determine the fluctuating pressure field [1]:

$\frac{\partial^{2}}{\partial x_{i}^{2}} p=-\frac{\partial}{\partial x_{i}} \frac{\partial}{\partial x_{j}}\left(u_{i} u_{j}\right)$.

Here, $p$ is the pressure divided by density, $u_{i}$ are the velocity components and the summation convention applies. From the normal component of the NavierStokes equations we get the following boundary condition for the pressure gradient at the walls:

$\frac{\partial p}{\partial x_{3}}=\nu \frac{\partial^{2} u_{3}}{\partial x_{3}^{2}}=-\nu \frac{\partial}{\partial x_{3}}\left(\frac{\partial u_{1}}{\partial x_{1}}+\frac{\partial u_{2}}{\partial x_{2}}\right)$.

The non-dimensional viscosity $\nu \equiv \nu_{0} /\left(D u_{0}\right)$ has the constant value $10^{-4}$ in this simulation. As a consequence of eqs (2) the pressure at some point is not a function of the local velocities only (which are zero at the walls) rather than of the total velocity field in the surroundings. As the viscosity is small, the normal gradient of $p$ given by eq. ( $2 b$ ) at the walls is small if compared to those values in the bulk of the flow. Therefore, the viscous sublayer does not affect the wall pressure considerably.

The finite difference analogue to eqs (2) has been solved at each time step using a non-iterative method based on fast Fourier transformation [1, appendix 4]. The present method is an extension of that developed by Deardorff [10]. It is the first of this kind to be applicable to an annulus.

Three different runs have been performed for this purpose; they are termed $\mathrm{K}_{3}, \mathrm{~K}_{4}$ (plane channel) and
Table 1 .

Parameters of runs $Z_{3}, Z_{3}^{\prime \prime}, K_{3}$ and $K_{4} \cdot{ }^{*}$

\begin{tabular}{|c|c|c|c|}
\hline & $\mathrm{Z}_{3}, \mathrm{Z}_{3}^{\prime \prime}$ & $\mathrm{K}_{3}$ & $\mathrm{~K}_{4}$ \\
\hline \multicolumn{4}{|c|}{ Length of periodicity in the } \\
\hline$x_{1}$ direction & $4 D$ & $4 D$ & $4 D$ \\
\hline$\varphi$ or $x_{2}$ directions & $2 \pi$ & $2 D$ & $2 D$ \\
\hline \multicolumn{4}{|c|}{ Number of grid points in the } \\
\hline$x_{1}$ direction & 32 & 64 & 64 \\
\hline$x_{2}$ direction & 64 & 32 & 32 \\
\hline$x_{3}$ direction & 16 & 16 & 32 \\
\hline Total & 32768 & 32768 & 65536 \\
\hline
\end{tabular}

* Grid spacing is equidistant in all directions except for $\mathrm{K}_{4}$ where the radial spacings vary between 0.018 and 0.042 .

Table 2.

Times over which the average is taken; unit: $D /\left(r_{\mathrm{w}_{0}} / \rho\right)^{1 / 2} .^{*}$

\begin{tabular}{llll}
\hline$Z_{3}$ & $Z_{3}^{\prime \prime}$ & $K_{3}$ & $K_{4}$ \\
\hline 1.776 & 4.865 & 0.920 & 4.069 \\
2.109 & & 1.338 & 4.163 \\
2.482 & & 1.791 & 4.255 \\
2.871 & & 2.242 & 4.353 \\
3.288 & & 2.684 & 4.451 \\
3.676 & & 3.140 & 4.549 \\
4.052 & & 3.591 & \\
4.440 & & 3.977 & \\
4.865 & & & \\
\hline
\end{tabular}

* $\mathrm{Z}_{3}$ and $\mathrm{K}_{3}$ started from random initial values and $\mathrm{K}_{4}$ from the result of $\mathrm{K}_{3}$ at time 3.977 by interpolation.

$Z_{3}$ (annulus). $Z_{3}^{\prime \prime}$ is a special result of $Z_{3}$ for one specific time. Additional runs denoted by $K_{1}, K_{2}, Z_{1}, Z_{2}$ and $Z_{4}$ have been considered for other purposes [1]. The present cases are preferred because they involve longer integration times and a large number of grid points. The parameters of these runs are given in table 1.

Instead of full time averaging, the averages (denoted by \langle\rangle$)$ are taken at fixed times over planes which are parallel to the walls. In addition, these values are averaged over at least six results at different times in the course of integration. The values of these times are listed in table 2. However, as can be seen from the results, there are still statistical fluctuations. The figures are unsmoothed reproductions of computer graphics so the statistical errors can be estimated. Some of the errors appear because the earlier results used in case $Z_{3}$ are not in the steady state. 


\section{Results}

\subsection{Velocity and pressure fluctuations}

Good agreement between numerical and experimental results for the r.m.s. (root mean square) values of fluctuating velocities and pressure have been found in the plane channel $[1,2,8]$. Fig. 1 shows the fluctuating velocities in an annulus. The general order of magnitude agrees with experimental results. (So far, experimental values are not available to the author for this particular value of $R_{2} / R_{1}$, but we may refer to the results of Lawn and Elliot [11] for $R_{2} / R_{1}=5.7$.)

Accidently, for case $Z_{3}$ the fluctuating velocities are higher close to the inner wall than to the outer wall, which shows that the steady state was not yet reached here. Case $Z_{3}^{\prime \prime}$, where we average over the results at the final time only, furnishes more reasonable results. The same effects can be seen for the r.m.s. values of the
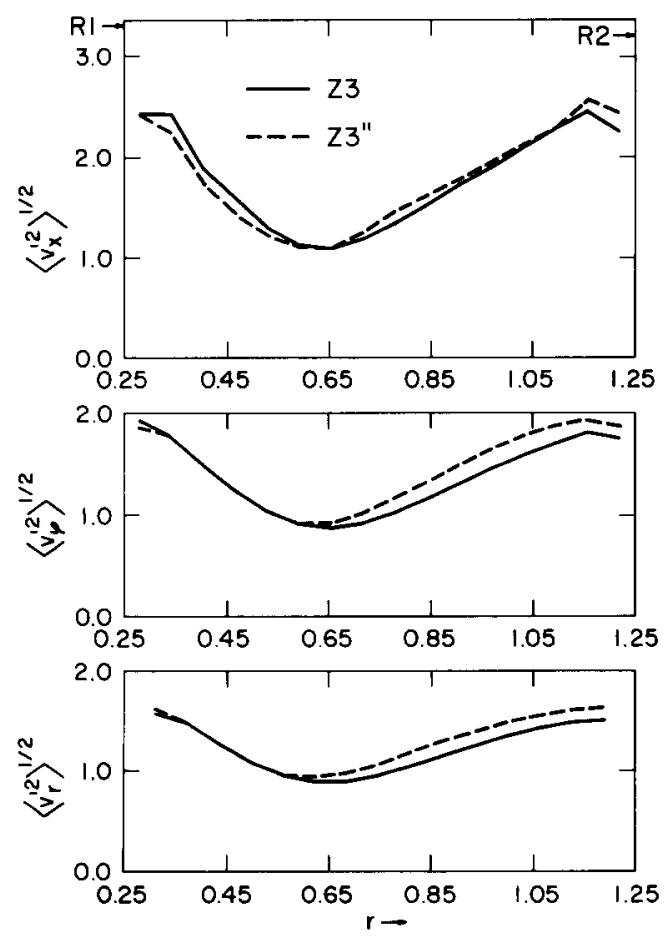

Fig. 1. R.m.s. values of fluctuating velocities normalized by the friction velocity in an annulus as a function of the radial coordinate. $Z_{3}$ is the average value over nine realizations, not all of which are in the steady state. $Z_{3}^{\prime \prime}$ is the average over the last realization. $\left\{v_{x}^{\prime}, v_{\phi}^{\prime}, v_{r}^{\prime}\right\}$ are the components of the fluctuating velocity vector in the $\{x, \varphi, r\}$ directions.
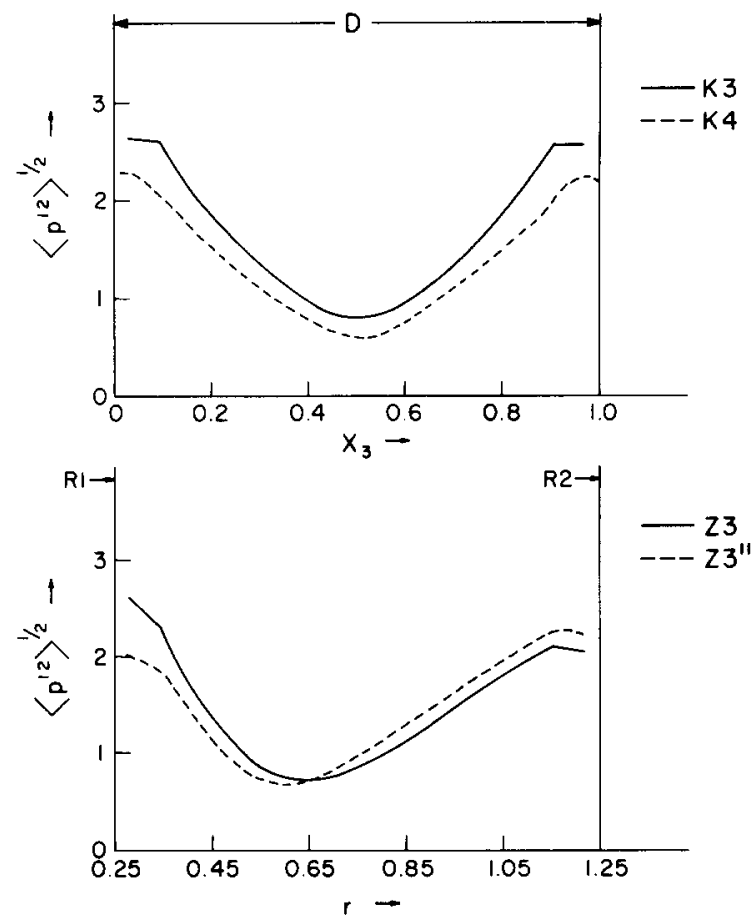

Fig. 2. R.m.s. values of the fluctuating pressure normalized by the mean wall shear stress in a plane channel $\left(\mathrm{K}_{3}\right.$ and $\left.\mathrm{K}_{4}\right)$ and an annulus $\left(Z_{3}, Z_{3}^{\prime \prime}\right)$. For the differences between $Z_{3}$ and $Z_{3}^{\prime \prime}$, see fig. 1 .

fluctuating pressure for cases $Z_{3}$ and $Z_{3}^{\prime \prime}$ in fig. 2. The corresponding results for the pressure fluctuations in the plane channel $\left(\mathrm{K}_{3}, \mathrm{~K}_{4}\right)$ show the expected sym. metry. The systematic differences between $K_{3}$ and $K_{4}$ cannot be explained satisfactorily, however.

The wall stresses have been found to be

\begin{tabular}{llll}
\hline & $\mathrm{Z}_{3}$ & $\mathrm{Z}_{3}^{\prime \prime}$ & $\mathrm{K}_{3}, \mathrm{~K}_{4}$ \\
\hline$\left\langle\tau_{\mathbf{w}_{1}}\right\rangle /\left\langle\tau_{\mathbf{w}_{\mathbf{0}}}\right\rangle$ & 1.24 & 1.31 & 1 \\
$\left\langle\tau_{\mathbf{w}_{2}}\right\rangle /\left\langle\tau_{\mathbf{w}_{0}}\right\rangle$ & 0.953 & 0.94 & 1 \\
\hline
\end{tabular}

The r.m.s. value of the wall pressure normalized with these wall stresses is

\begin{tabular}{lllll}
\hline & & $\mathrm{Z}_{3}^{\prime \prime}$ & $\mathrm{K}_{3}$ & $\mathrm{~K}_{4}$ \\
\hline$\left\langle p^{2}\right\rangle^{1 / 2} /\left\langle\tau_{\mathrm{w}}\right\rangle$ & $r=R_{1}$ & 1.52 & 2.65 & 2.28 \\
& $r=R_{2}$ & 2.35 & 2.57 & 2.24 \\
\hline
\end{tabular}


The result $\left\langle p^{2}\right\rangle^{1 / 2} /\left\langle\tau_{w}\right\rangle \approx 2.4 \pm 0.2$ for the plane channel is in accordance with a large number of measurements [12]. This ratio is remarkably smaller at the inner wall of the annulus, however.

\subsection{Pattern of the wall pressure fluctuations}

Figures 3 and 4 give isometric contour line plots of the pressure fluctuations at the walls. They give us an impression of the attainable resolution and the general structure of the wall pressure field. The tick marks indicate the grid lines and the periodical boundary conditions are evident. The minimum and maximum values of the pressure fluctuations are of the order $\pm 10\left\langle\tau_{w_{0}}\right\rangle$. The following conclusions, which will be seen from the evaluated correlation functions quantatively (see below), can be drawn.

The patterns show no clear elongation in the axial or azimuthal directions especially for the plane channel. Some elongation in the azimuthal direction does exist at the outer wall of the annulus whereas some axial elongation seems to exist at the inner wall in this geometry. However, the latter are connected with the peaks of the pressure fluctuations mainly and it is possible that they are a consequence of the high resolution in the azimuthal direction. No significant differences can be found between case $K_{3}$ and $K_{4}$. From corresponding pictures for the velocity components (in the bulk of the flow) $[1,10]$, we know that there exists a clear elongation of the axial velocity components and virtually no elongation for the other components. This suggests that the structure of the wall pressure is determined by the radial velocity components as one would expect.

\subsection{Spatial pressure correlations}

We consider the normalized wall pressure correlation function

$$
\begin{aligned}
& P P(x, \varphi, r) \equiv\left\langle p\left(z_{1}, z_{2}, r\right) p\left(z_{1}+x, z_{2}+\varphi, r\right)\right\rangle /\left\langle p^{2}\right\rangle, \\
& \quad r=R_{1}, R_{2} .
\end{aligned}
$$

Here the averaging operator \langle\rangle includes integration over all $z_{1}, z_{2}$. For a plane channel we use a corresponding definition for $P P\left(x_{1}, x_{2}, r\right), r=R_{1}, R_{2}$. Fig. 5 shows this correlation as a function of the azimuthal coordinate $\varphi$ for $Z_{3}$. Fig. 6 shows the corresponding result $P P\left(0, x_{2}, r\right)$ for the plane channel $\left(\mathrm{K}_{3}\right)$. The axial variations are given in fig. 7 .

Characteristic length scales for these correlations are those separation values $l_{1}$ and $l_{2}$ (or $\varphi_{2}=l_{2} / r$ ) for which $P P\left(l_{1}, 0, r\right)$ or $P P\left(0, l_{2}, r\right)$ are equal to 0.5 . The
Fig. 3. Contour plots of the fluctuating pressure at the inner $(P W 1)$ and outer $(P W 2)$ wall of an annulus (case $\left.Z_{3}^{\prime \prime}\right)$. Dashed lines indicate negative values. The numbers beside are the values of the pressure at the corresponding contour lines in units of $\left\langle\tau_{\mathbf{W}_{0}}\right\rangle$. 
K3

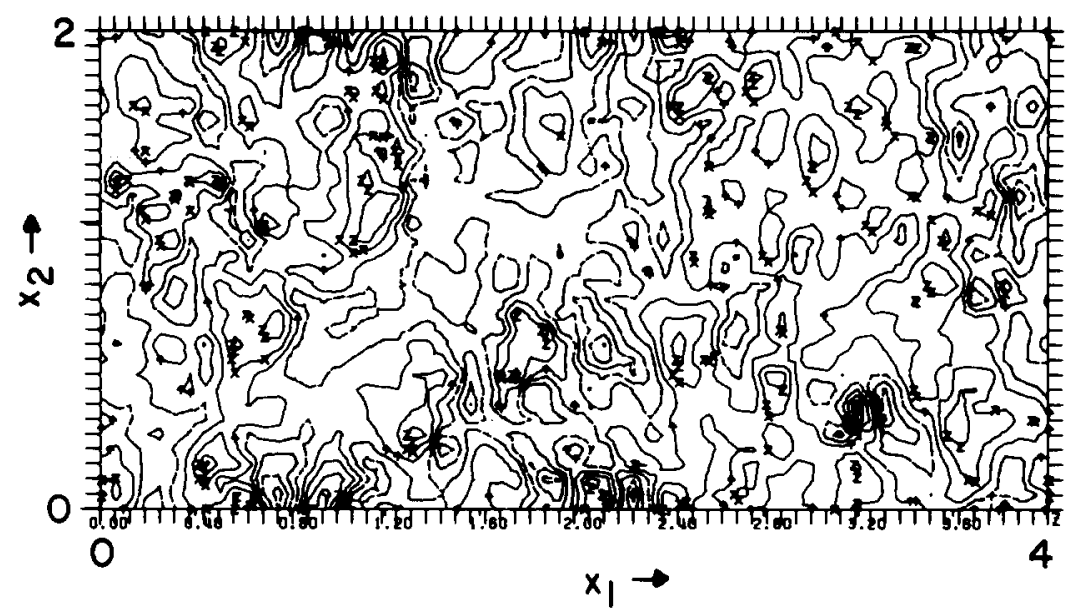

PW1

- -0.102E 02

$\triangle-0.803 E$ O1

$+-0.585 E 01$

$x-0.368 E 01$

$-0.151 E$ or

- 0.6665 oo

$\times 0.284 E 01$

$=0.501 E$ o1

- $0.719 E 01$

- $0.936 E$ o1

\section{$\mathrm{K} 4$}

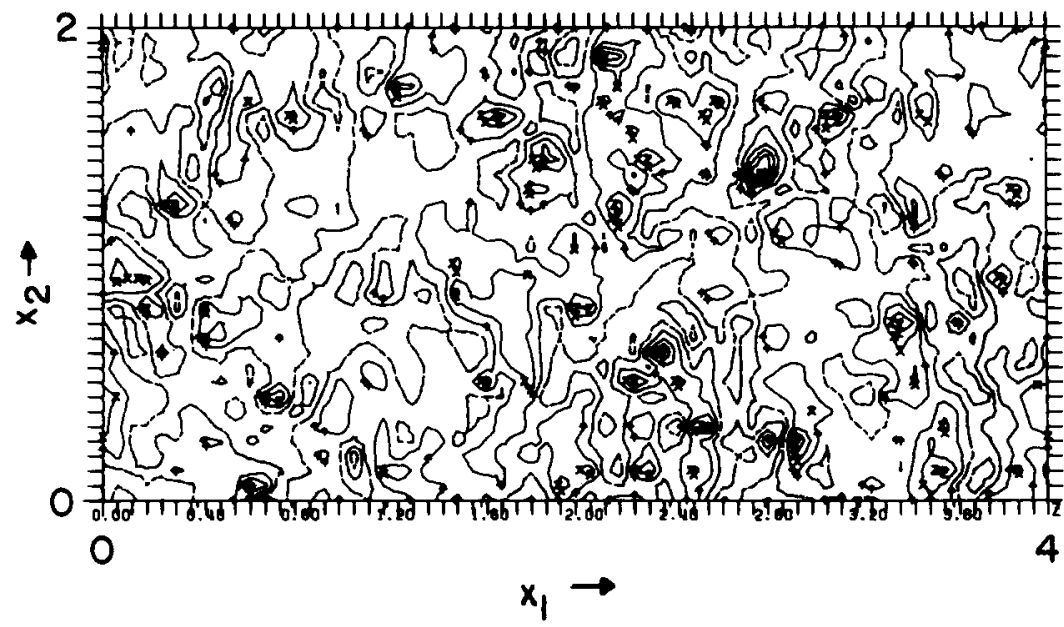

PW1

$0-0.948601$

$4-0.733 E 01$

$+-0.516 E 01$

$x-0.304 E 01$

- -0.890e 00

- $0.126 E 01$

$\times 0.341 E$ O1

> 0.555501

- $0.770 \mathrm{E} 01$

D $0.985 E 01$

Fig. 4. Same as fig. 3 for the plane channel (cases $\mathrm{K}_{3}$ and $\mathrm{K}_{4}$ ) at $r=R_{1}$; similar patterns have been found for $r=R_{2}$.

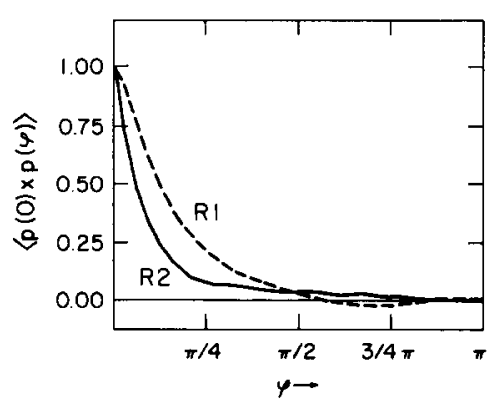

Fig. 5. Normalized pressure correlations for an annulus as a function of the azimuthal coordinate $\varphi$ at the inner $\left(R=R_{1}\right)$ and outer $\left(R=R_{2}\right)$ walls (case $\left.\mathrm{Z}_{3}\right)$.

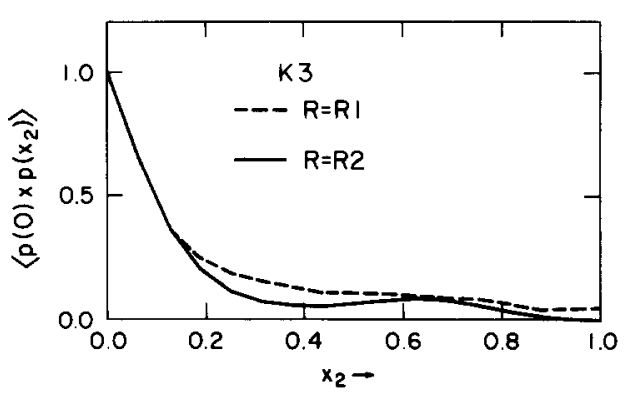

Fig. 6. Normalized pressure correlations for a plane channel as a function of the 'azimuthal' coordinate $x_{2}$ at both walls. 

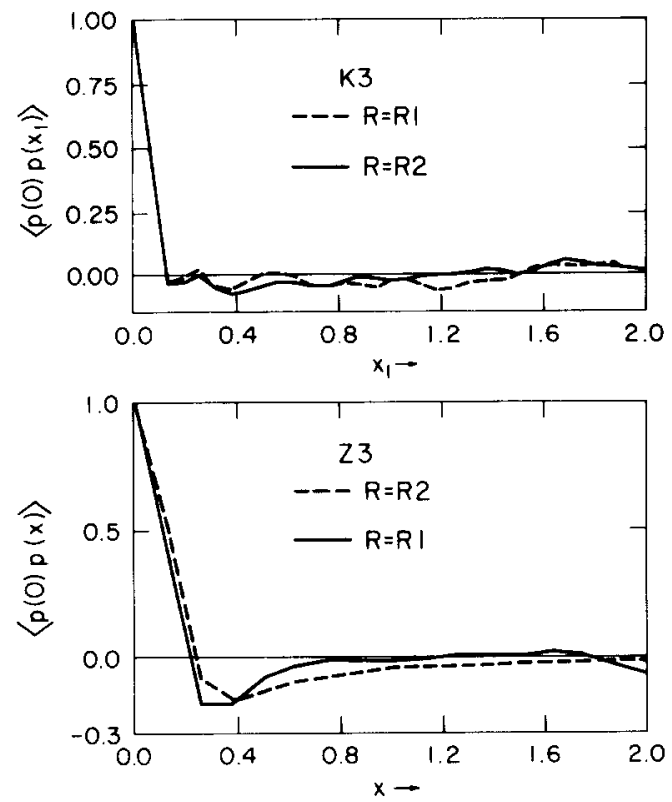

Fig. 7. Normalized pressure correlations for a plane channel $\left(\mathrm{K}_{3}\right)$ and an annulus $\left(\mathrm{Z}_{3}\right)$ as a function of the axial coordinate at the walls.

Table 3.

Characteristic length scales of correlation functions $I_{1}$ and $l_{2}$ in the axial and azimuthal directions.

\begin{tabular}{lllc}
\hline Case & $l_{1} / D$ & $l_{2} / D$ & $\varphi_{2} \equiv l_{2} / r$ \\
\hline$Z_{3}, r=R_{1}$ & 0.11 & 0.09 & $0.37 \approx 22^{\circ}$ \\
$Z_{3}, r=R_{2}$ & 0.14 & 0.22 & $0.18 \approx 10^{\circ}$ \\
$\mathrm{K}_{3}$ & 0.075 & 0.1 & - \\
\hline
\end{tabular}

values of these length scales are given in table 3 . We see that $l_{1}$ and $l_{2}$ are of the same magnitude as estimated from the flow patterns (figs 3 and 4 ). The length scales $l_{2}$ are different for the inner and outer walls of the annulus and not proportional to the radius. Although we cannot expect a high accuracy for these values, because they are of the same order as the finite difference grid spacings, we must conclude that the wall pressure correlation functions at the inner and outer walls are not similar. So, the replacement of measurements at the inner wall by those at the outer wall [5] or, even more so, the use of a pipe [13] instead of an annulus cannot be justified.

From fig. 5 we see that there is virtually no correlation between the pressure at two points which are diametrically opposed to each other. The correlation
$P P(0, \varphi, r)$ is already very close to zero for $\varphi \geqslant \pi / 2$. This result can be assumed to be generally valid for all annuli with $R_{2} / R_{1} \leqslant 5$. We therefore obtain the relationship

$$
\begin{aligned}
& \left\langle[ p ( z _ { 1 } , z _ { 2 } , r ) - p ( z _ { 1 } , z _ { 2 } + \pi , r ) ] \left[ p\left(z_{1}, z_{2}+\varphi, r\right)\right.\right. \\
& \left.\left.\quad-p\left(z_{1}, z_{2}+\pi+\varphi, r\right)\right]\right\rangle \approx 2\left\langle p\left(z_{1}, z_{2}, r\right) p\left(z_{1}, z_{2}+\varphi, r\right)\right\rangle, \\
& 0 \leqslant|\varphi| \leqslant \pi / 2, R_{2} / R_{1} \leqslant 5,
\end{aligned}
$$

where the former quantity has been measured by Gorman [5]. On the other hand, Appelt et al. [7] measured $\left\langle p_{1}^{2}\right\rangle,\left\langle p_{2}^{2}\right\rangle$ and $\left\langle\left(p_{1}-p_{2}\right)^{2}\right\rangle$ in an annulus $\left(R_{2} / R_{1}=2.77\right)$ where $p_{1}$ and $p_{2}$ were the fluctuating pressures on opposite sides of the internal cylinder. From their measurements the correlation coefficient $P P\left(0, \pi, R_{1}\right)$ can be calculated from

$P P\left(0, \pi, R_{1}\right)=1-2\left\langle\left(p_{1}-p_{2}\right)^{2}\right\rangle /\left(\left\langle p_{1}^{2}\right\rangle+\left\langle p_{2}^{2}\right\rangle\right)$.

This results in the values 0.476 for the loop with a settling chamber and 0.949 without a settling chamber. Both values differ greatly from zero. This indicates that the pressure fluctuations in this loop are not caused by turbulence alone, but also by external sources which give rise to pressure waves travelling through the channel in an axial direction. The above values of $P P\left(0, \pi, R_{1}\right)$ furnish the relative fraction of the contribution of these pressure waves to the pressure fluctuations.

The axial correlation functions (fig. 7) show some oscillations around zero for large values of the separation distance $x_{1}$ in case $K_{3}$ and at least one overshoot in case $Z_{3}$ (fig. 7). Oscillations of the same order of magnitude have been found by Gorman [5] in an annulus $\left(R_{2} / R_{1}=1.67\right)$ and even larger ones by Bakewell [13] for a pipe, but they did not appear in a boundary layer flow [12]. Hence, it must be concluded that these oscillations are strongly geometry-dependent and equations based on measurements for one specific geometry, such as those used by Reavis [4], are not sufficient. No significant oscillations are to be seen in the functions $P P(0, \varphi, r)$, which is as found experimentally.

All axial correlation values are very close to zero for an axial separation of $x=2 D$. The periodicai boundary condition in the axial direction with a length of periodicity equal to $4 D$ (see table 1) is therefore acceptable for this kind of investigation. 


\subsection{Forces acting on the outer and inner cylinders $\left(Z_{3}\right)$}

The components of the forces per unit axial length on the cylinders in two orthogonal directions caused by the pressure fluctuations are given by

$F X(x, r)=\int_{0}^{2 \pi} p(x, \varphi, r)(\cos \varphi) r \mathrm{~d} \varphi$

and

$F Y(x, r)=\int_{0}^{2 \pi} p(x, \varphi, r)(\sin \varphi) r \mathrm{~d} \varphi$.

To give an idea of the behavior of these forces we show one of their realizations in fig. 8 above $\left(Z_{3}^{\prime \prime}\right)$. The average power spectrum $S(K)$ of both components at this time and corresponding ones for other times $\left(Z_{3}\right)$ are shown below in a linear and again in a logarithmic plot. $S(K)$ is defined by

$S(K)=\left\langle U(K) U(K)^{*}\right\rangle$,
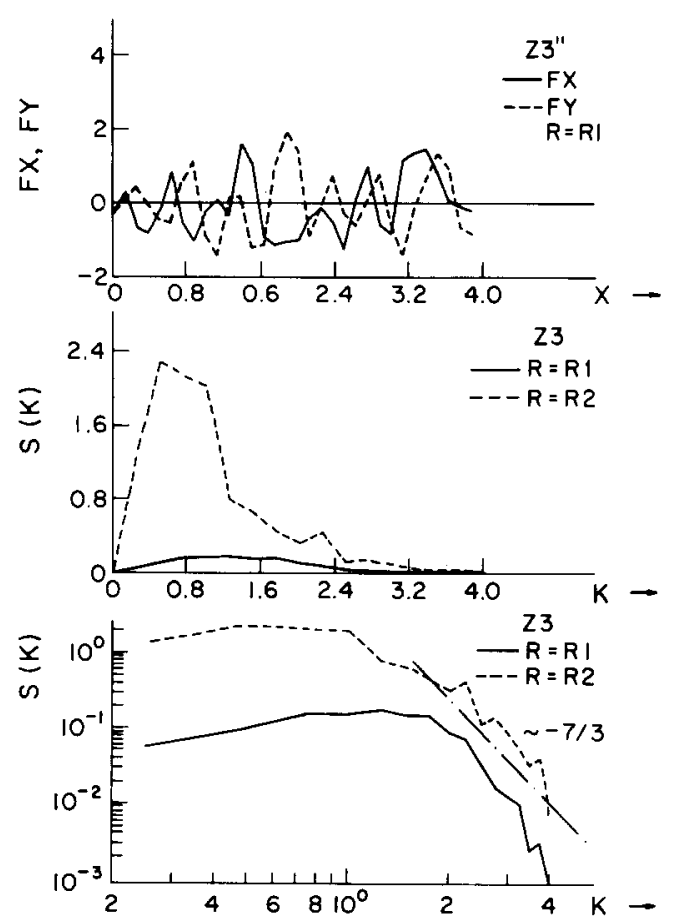

Fig. 8. Top: example of the variation of the forces acting on the inner cylinder of the annulus. Center and bottom: power spectrum of the forces at both walls as a function of the wave number $K$. The forces are measured in units of $\left(D\left\langle\tau_{\mathbf{W}, 0}\right)\right)$. where $U(K)$ is the Fourier transform of $F(x)$ defined by a finite difference counterpart of

$U(K)=\int_{-\infty}^{\infty} F(x) \exp (-\mathrm{i} 2 \pi K x) \mathrm{d} x$

in which $U(K)^{*}$ is the conjugate complex value of $U(K), K$ is the wave number and $F(x)$ is either $F X(x)$ or $F Y(x)$. From the linear plot we find $S(K)$ to be zero for $K=0$, which is the consequence of the zero mean of the forces and the one-dimensional nature of $F(x)$. From the logarithmic plot we see that the spectrum follows a $-7 / 3$ power law over some region of the larger wave numbers which agrees with the spectrum of the pressure fluctuations [9]. The spectra reach their maximum values at a wave number $K \approx 1 / D$. The correlation function

$$
\begin{aligned}
& F F(x, r)=\frac{1}{2}\langle F X(z, r) F X(z+x, r)+F Y(z, r) \\
& \quad \times F Y(z+x, r)\rangle\left\langle\left\langle F^{2}\right\rangle\right.
\end{aligned}
$$

is given for $r=R_{1}, R_{2}$ in fig. 9 . The value
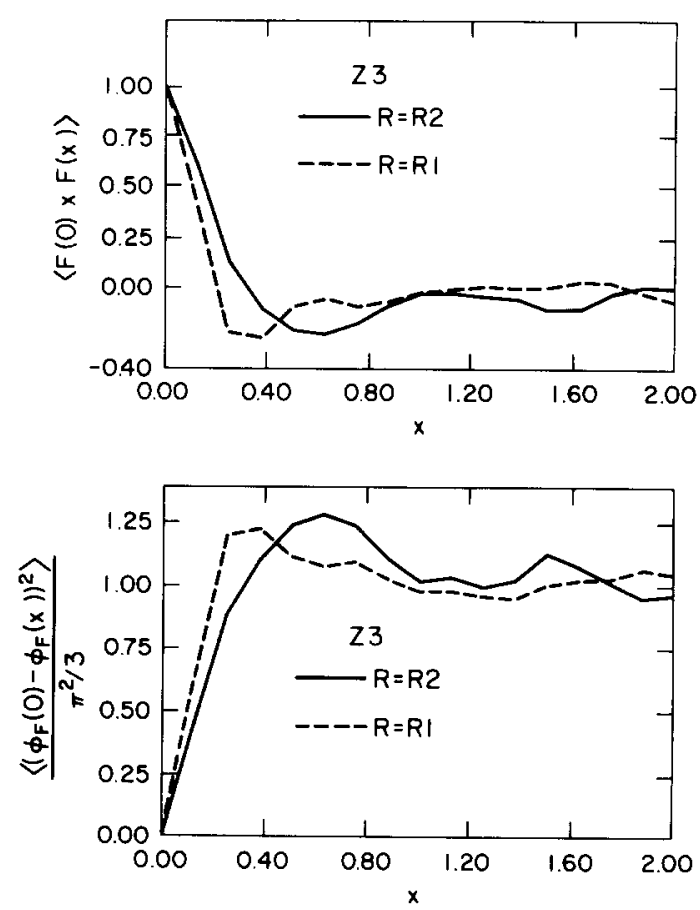

Fig. 9. Normalized force correlation as a function of the axial direction (above) and angular correlations between the directions $\Phi_{F}$ of the force vector at different positions separated axially (below) for an annulus at both walls. 


$$
\left\langle F^{2}\right\rangle=\frac{1}{2}\left\langle F X(z, r)^{2}+F Y(z, r)^{2}\right\rangle
$$

was found to be as follows (unit $\left.\left(\left\langle\tau_{\mathrm{w}_{0}}\right\rangle D\right)^{2}\right)$ :

\begin{tabular}{rcr}
\hline $\mathrm{Z}_{3}$ & $\mathrm{Z}_{3}^{\prime \prime}$ & \\
\hline 1.2 & 0.68 & $r=R_{1}$ \\
10.3 & 21.3 & $r=R_{2}$
\end{tabular}

From these values we are able to compute a 'ratio of the effective diameter to the actual diameter $\psi_{\mathrm{D}}$ ' $[4,5]$ which is defined by

$\psi_{\mathrm{D}}=\left\langle F^{2}\right\rangle^{1 / 2} /\left(2 r\left\langle p^{2}\right\rangle^{1 / 2}\right)$.

We find

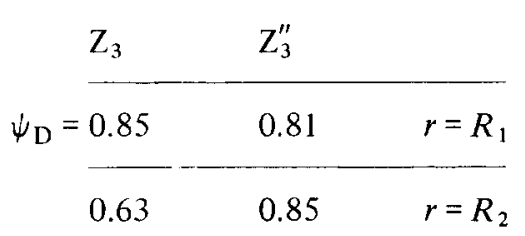

These values are about the same for both walls. This must be assumed to be accidental. The values are rather high. As we expect $\psi_{\mathrm{D}}$ to be zero for $R_{2} / R_{1} \rightarrow 1$ as well as $R_{2} / R_{1} \rightarrow \infty$, these values found for $R_{2} / R_{1}=5 \mathrm{might}$ be very close to a maximum value.

The lower part of fig. 9 describes the changes in the direction of the forces. We consider the angular direction $\phi_{\mathrm{F}}$ of the total force vector

$$
\phi_{\mathrm{F}}=\arctan [F X(x) / F Y(x)]+\pi, \quad 0 \leqslant \phi_{\mathrm{F}} \leqslant 2 \pi
$$

and calculate the correlation functions

$\phi \phi(x, r)=\left\langle Q^{2}\right\rangle / N_{Q}$,

where $Q$ is the smallest amount of the differences between the angles $\phi_{\mathrm{F}}$ at two axial positions

$$
\begin{gathered}
Q(x)=\min \left\{\left|\Phi_{\mathrm{F}}(z, r)-\Phi_{\mathrm{F}}(z+x, r)\right|,\right. \\
\left.2 \pi-\left|\Phi_{\mathrm{F}}(z, r)-\Phi_{\mathrm{F}}(z+x, r)\right|\right\} .
\end{gathered}
$$

For large values of $x$ the directions of the forces are independent and so the probability function of $Q$ is

$$
P(Q)=(1 / \pi) Q, \quad 0 \leqslant Q \leqslant \pi .
$$

Therefore, we normalize with

$N_{Q} \equiv\left\langle Q(\infty)^{2}\right\rangle=\int_{0}^{\pi} Q P(Q) \mathrm{d} Q=\pi^{2} / 3$.

In fact, we see from fig. 9 that the value thus normalized of $\phi \phi$ approaches unity for large $x$.

We find $[1-\phi \phi(x, r)]$ to be very similar to $F F(x, r)$. For both we find a correlation length $l_{1}$ defined by $F F\left(l_{1}, r\right) \approx \phi \phi\left(l_{1}, r\right)=0.5$ which is roughly equal to that $l_{1}$ which was found for the axial pressure correlation functions in the $\mathrm{Z}_{3}$ case. As $F F$ includes the integration over both $\varphi$ and $x$, the similarity between $F F(x, r)$ and $P P(x, 0, r)$ provides a solid basis for the assumption that $P P(x, \varphi, r)$ can be separated in space according to

$P P(x, \varphi, r) \approx P P(x, 0, r) P P(0, \varphi, r)$.

This assumption was made earlier $[4,5]$ and the results of the present work may be taken as confirmation.

\section{Conclusions}

It has been shown that fluctuations of the wall pressure can be calculated by means of the finite difference scheme $[1,8]$, which furnishes turbulent channel flows in time and space. The accuracy of the results is limited by the size of the finite difference grid spacings, the departure from steady state, and statistical fluctuations due to finite sampling. A variation of the parameter $R_{2} / R_{1}$, which took the value $5 / 1$ in this study, is highly desirable, but about $20 \mathrm{hr}$ of computer time on an IBM 370/165 are needed to run one case with 32768 grid points, as in case $Z_{3}$.

The r.m.s. values of the wall pressure fluctuations generated by turbulence are about 2.4 times the wall stress in a plane channel and on the outer wall of the annulus, but this ratio is much smaller at the inner wall of the annulus. From the flow patterns and the pressure correlation functions evaluated at the outer and inner walls of the annulus and at the walls of the plane channel we conclude that the axial correlation functions are similar in all these cases and show the oscillations found elsewhere in channel flows. The azimuthal correlation functions are not similar in that the characteristic length scales are smaller at the inner wall than at the outer wall, but not in the same ratio as $R_{1} / R_{2}$. We 
found that $P P(0, \varphi, r)$ is zero at the walls for $\varphi>\pi / 2$ and $R_{2} / R_{1} \leqslant 5$. This result can be used to discriminate pressure fluctuations generated by turbulence from those generated by the test loop in experimental investigations.

By integrating the pressure field we were able to compute the resultant forces acting on the inner and outer cylinders of the annulus. Spectra and correlation functions are shown. The effective diameter, eq. (10), was found to be about 0.8 the actual diameter for both walls. The shape of the correlation functions is very close to that of the axial pressure correlation function.

To the best of our knowledge this is the first approach to an evaluation of the wall pressure and force correlation functions without specific experimental support. The method of direct numerical simulation of turbulent flows has been found to be applicable in this case.

\section{Nomenclature}

\begin{tabular}{|c|c|}
\hline$D$ & $\begin{aligned}= & \text { distance between the walls } \\
& \left(D=R_{2}-R_{1}\right)\end{aligned}$ \\
\hline$F$ & $=$ force per unit length divided by density \\
\hline$F F$ & $=$ force correlation function, eq. (9) \\
\hline$F X$ & $=$ force component in $x_{2}$ direction \\
\hline$F Y$ & $=$ force component in $x_{3}$ direction \\
\hline$K$ & $=$ wave number \\
\hline$l_{1}$ & $\begin{aligned}= & \text { correlation length scale in } x, x_{1} \\
& \text { direction }\end{aligned}$ \\
\hline$l_{2}$ & $\begin{aligned}= & \text { correlation length scale in } \varphi, x_{2} \\
& \text { direction }\end{aligned}$ \\
\hline$p$ & $\begin{array}{l}=\text { fluctuating pressure divided by } \\
\text { density }\end{array}$ \\
\hline $\left.\begin{array}{l}P P(x, \varphi, r) \\
P P\left(x_{1}, x_{2}, r\right)\end{array}\right\}$ & $=$ pressure correlation function, eq. (3) \\
\hline$r$ & $=$ radial coordinate \\
\hline$R_{1}$ & $=$ radius of the inner wall \\
\hline$R_{2}$ & $=$ radius of the outer wall \\
\hline$S(K)$ & $=$ power spectrum of the forces, eq. (7) \\
\hline$u_{0}=\left(\left\langle\tau_{\mathrm{w}_{0}}\right\rangle\right)^{1 / 2}$ & $=$ friction velocity \\
\hline$\left\{x_{1}, x_{2}, x_{3}\right\}$ & $\begin{aligned}= & \text { Cartesian coordinates; } x_{1} \text { axial, } x_{2} \\
& \text { 'azimuthal', } x_{3}=r \text { radial }\end{aligned}$ \\
\hline$\{x, \varphi, r\}$ & $\begin{array}{l}=\text { cylindrical coordinates; } x \text { axial, } \varphi \\
\text { 'azimuthal', } r \text { radial }\end{array}$ \\
\hline$z_{1}, z_{2}$ & $\begin{aligned}= & \text { dummy variables for integration in } \\
& \text { the axial and azimuthal directions }\end{aligned}$ \\
\hline
\end{tabular}

$$
\begin{aligned}
& \nu_{0} \quad=\text { kinematic viscosity } \\
& \nu=\nu_{0} /\left(u_{0} D\right)=\text { non-dimensional viscosity } \\
& \left\langle\tau_{\mathbf{w}_{0}}\right\rangle \quad=\text { mean wall stress divided by density, } \\
& \text { eq. (1) } \\
& \left\langle\tau_{w_{1}}\right\rangle=\text { time mean wall stress divided by } \\
& \text { density at the inner wall }\left(r=R_{1}\right) \\
& =\text { time mean wall stress divided by } \\
& \text { density at the outer wall }\left(r=R_{2}\right) \\
& =\text { azimuthal coordinate } \\
& =\text { angle between the force vectors at } \\
& \text { two points, eq. (11) } \\
& =\text { angular correlation function of the } \\
& \text { forces, eq. (12). }
\end{aligned}
$$

\section{References}

[1] U. Schumann, Ein Verfahren zur direkten numerischen Simulation turbulenter Strömungen in Platten- und Ringspaltkanälen und über seine Anwendung zur Untersuchung von Turbulenzmodellen, Dissertation Fak. für Maschinenbau, Univ. Karlsruhe, KFK-1854 (1973).

[2] U. Schumann, Results of a numerical simulation of turbulent channel flows, ANS International Meeting on Reactor Heat Transfer, M. Dalle Donne (Ed.) Karlsruhe, Germany, 1973, Paper 40.

[3] J. Kadlec and K.D. Appelt, Flow induced rod vibrations of fast reactor subassemblies, Nucl. Eng. Des. 14 (1970) 136-150.

[4] J.R. Reavis, Vibration correlation for maximum fuelelement displacement in parallel turbulent flow, Nucl. Sci. Eng. 38 (1969) 63-69.

[5] D.J. Gorman, The role of turbulence in the vibration of reactor fuel elements in liquid flow, AECL-3371 (1969).

[6] M. Dubourg, R. Assedo, C. Cauquelin, C. Berriaud and M. Livolant, Model experimentation and analysis of flow-induced vibrations of PWR internals, Nucl. Eng. Des. 27 (1974) 315-333.

[7] K.D. Appelt, J. Kadlec, W. Krüger, E. Ohlmer and R. Schwemmle, Experimental investigation of loop caused influences on parallel flow-induced vibration of fuel pins, KFK 1385 (1971).

[8] U. Schumann, Subgrid scale model for finite difference simulations of turbulent flows in plane channels and annuli (to be published).

[9] J.W. Deardorff, Three-dimensional numerical study of turbulence in an entraining mixed layer, Boundary-Layer Meteorol. 7 (1974), 199-226.

[10] J.W. Deardorff, A numerical study of three-dimensional turbulent channel flow at large Reynolds numbers, J. Fluid Mech. 41 (1970) 453-480.

[11] C.J. Lawn and C.J. Elliot, Fully developed turbulent flow through concentric annuli, C.E.G.B. Report, RD/ B/N1878 (1971). 
[12] W.W. Willmarth and C.E. Wooldridge, Measurement of the fluctuating piessure at the wall beneath a thick turbulent boundary layer, J. Fluid Mech. 14 (1962) $187-$ 210; corrigendum: J. Fluid Mech. 21 (1965) 107-109.

[13] H.P. Bakewell Jr, Narrow-band investigations of the longitudinal space-time correlation function in turbulent airflow, J. Acoust. Soc. Amer. 36 (1964) 146-148.
Note added in proof: Further references to experimental results and a theory to compute wall pressure statistics from velocity statistics are given in: R.L. Panton and J.H. Linebarger. Wall pressure spectra calculations for equilibrium boundary layers, J. Fluid Mech. 65 (1974) 261-287. 